\title{
Chest Computed Tomography and Lung Ultrasound Findings in COVID-19 Pneumonia: A Pocket Review for Non-radiologists
}

\author{
Davide Pata ${ }^{1 *}$, Piero Valentini ${ }^{1,2}$, Cristina De Rose ${ }^{1}$, Rita De Santis ${ }^{1}$, Rosa Morello ${ }^{1}$ and \\ Danilo Buonsenso ${ }^{2,3}$ \\ ${ }^{1}$ Istituto di Pediatria, Università Cattolica del Sacro Cuore, Rome, Italy, ${ }^{2}$ Dipartimento Scienze della salute della donna, del \\ bambino e di sanità pubblica, Fondazione Policlinico Universitario Agostino Gemelli IRCCS, Rome, Italy, ${ }^{3}$ Istituto di \\ Microbiologia, Università Cattolica del Sacro Cuore, Rome, Italy
}

OPEN ACCESS

Edited by:

Mehdi Mirsaeidi,

University of Miami, United States

Reviewed by:

Romeo Chira,

Iuliu Haţieganu University of Medicine

and Pharmacy, Romania

Xiaojiong Jia

Harvard Medical School,

United States

*Correspondence:

Davide Pata

davide.pata01@gmail.com

Specialty section:

This article was submitted to

Pulmonary Medicine,

a section of the journal

Frontiers in Medicine

Received: 14 April 2020

Accepted: 18 June 2020

Published: 26 June 2020

Citation:

Pata $D$, Valentini P, De Rose $C$,

De Santis $R$, Morello $R$ and

Buonsenso D (2020) Chest Computed

Tomography and Lung Ultrasound Findings in COVID-19 Pneumonia: A

Pocket Review for Non-radiologists. Front. Med. 7:375

doi: 10.3389/fmed.2020.00375
COVID-19 is an infectious disease that has quickly spread worldwide, causing a pandemic. The main clinical manifestation is pneumonia. The most important test for the diagnosis is represented by RT-PCR, but, given the limited sensitivity, further radiological examinations are necessary. We reviewed the literature to highlight the typical manifestations and advantages of chest computed tomography and lung ultrasound in COVID-19 pneumonia in order to assist clinical researchers in the management of this disease.

Keywords: COVID-19, chest computed tomography, lung ultrasound, SARS-CoV-2, diagnosis

\section{INTRODUCTION}

COVID-19 (COronaVIrus Disease 2019) is an infectious disease caused by a novel Coronavirus called SARS-CoV-2. Although initially described in Wuhan (1), the disease quickly spread worldwide, and the World Health Organization (WHO) defined COVID-19 as a pandemic on March 11th, 2020 ${ }^{1}$. On April 8th, 1,353,361 cases had been confirmed from all over the world (2).

The incubation period is between 1 and 14 days and averages 3-7 days (3). The common and mild symptoms are fever, fatigue, cough, pharyngitis, myalgia, arthralgia, anosmia, and dysgeusia (4-6). However, the most important clinical manifestation of COVID-19 is pneumonia, which may be complicated by acute respiratory distress syndrome (ARDS), leading, in some cases, to acute respiratory failure and exitus $(7,8)$.

The main test for the diagnosis of SARS-CoV-2 infection is the real-time reverse transcription polymerase chain reaction (RT-PCR), which is performed by nasopharyngeal swab. However, given the high rate of false negative results (9), several authors suggested the routine use of chest computed tomography in case of COVID-19 suspicion (CT) $(10,11)$. Fang et al. reported that CT scans have a sensitivity of $98 \%$ (12). However, Raptis et al. pointed out a series of bias errors and assumed a lower sensitivity (13). Another study reported that CT had limited sensitivity especially in the early stages of the disease: in the first 2 days after the onset of symptoms, $56 \%$ of patients had normal findings, and chest CT can therefore not be used to exclude SARS-CoV-2 infection (14). In addition, this test was used in subsequent monitoring (15).

Recent studies established that Lung Ultrasound (LUS) is a reliable technique in the diagnosis of lung diseases (16-19), even during pregnancy (20). As a result, a number of manuscripts were produced on the use of pulmonary Lung Ultrasound (LUS) during the COVID-19 epidemic.

${ }^{1}$ Available online at: https://www.who.int/dg/speeches/detail/who-director-general-s-opening-remarks-at-the-mediabriefing-on-covid-19-11-march-2020 (accessed April 04, 2020). 
Therefore, we performed a literature review on the use of CT and LUS in COVID-19 pneumonia in order to assist nonradiologists involved in the forefront of COVID-19.

\section{CT FINDINGS}

In their work, Shi et al. reported that all patients had pathological CT scans. The abnormalities can be seen in all lung segments and their number is directly related to disease severity. The most frequently reported distribution pattern is bilateral lung involvement (79\%), while peripheral and diffuse distributions are rarer, 54 and $44 \%$, respectively (21).

The typical CT pattern is characterized by bilateral distribution of ground glass opacities (GGO) with or without consolidations in posterior and peripheral lung fields $(22,23)$ (Figures 1, 2):

- GGO refers to areas of misty pulmonary opacity with conservation of parenchymal architecture, caused by the thickening of the alveolar septa due the inflammatory process

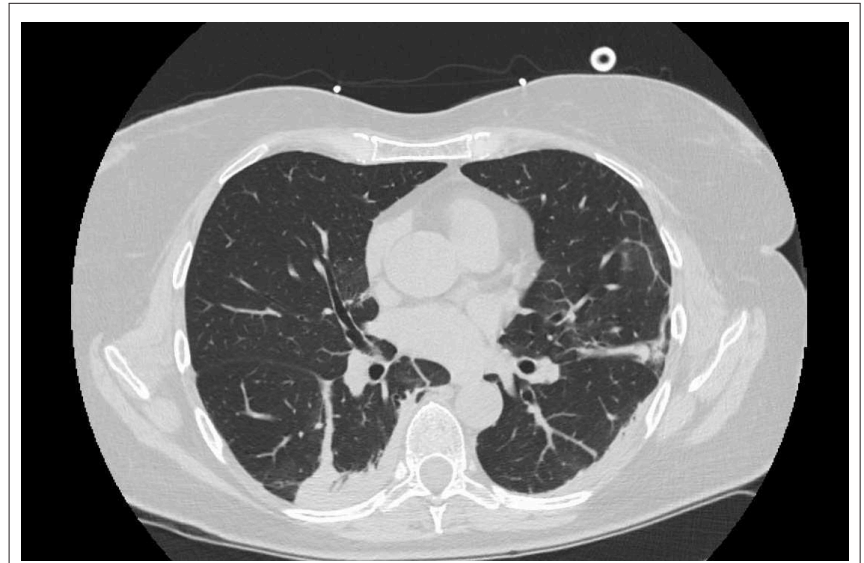

FIGURE 1 | Typical CT findings in patients with COVID-19 pneumonia. Peripheral ground glass lesion with consolidations and bronchiectasis.

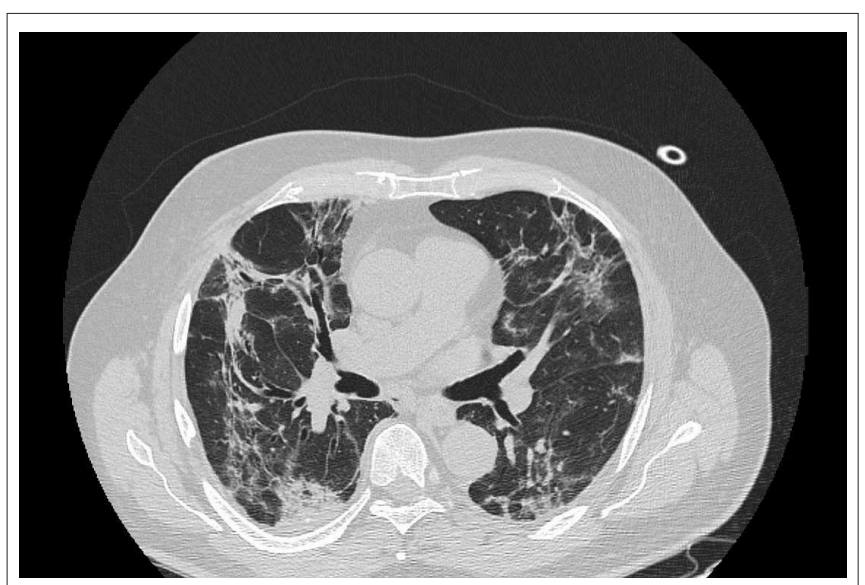

FIGURE 2 | Typical CT findings in patients with COVID-19 pneumonia. Consolidations and GGO, associated with bronchiectasis and crazy paving pattern.
(24). In COVID-19 pneumonia, unilateral or bilateral GGO are the most common CT findings (25-28)

- Reticular pattern refers to the presence of several linear opacities that give the appearance of a network. It is due to the interstitial thickening (24). Reticular pattern is the most frequent pattern in COVID-19 patients after GGO $(21,26,29)$

- Crazy paving pattern is defined as thickened interlobular septa superimposed on an area of GGO and is due to alveolar proteinosis (24). It indicates the transition to a progressive stage of COVID-19 pneumonia (30)

- Consolidation is an area of increased density with the elimination of normal lung parenchymal architecture. Air is replaced by fluids or cells, while vascular structures and bronchial walls are no longer recognizable (24). Consolidation is usually described in COVID-19 patients (28-30) and is another sign of disease progression (30)

- Pleural abnormalities, such as thickening and effusion, are less frequently described in COVID-19 pneumonia and could be a poor prognostic sign (21)

- Airway abnormalities include bronchiectasis and bronchial wall thickening. They are due to inflammatory damage (24) and are described in the most severe pneumonia (21)

- Air bronchogram is the direct visualization of an air-filled bronchus in the context of an opacity (24). Indeed, in COVID19 the bronchus is filled of highly viscous mucus instead of air (31)

- Fibrosis is defined as the replacement of normal tissue into scar tissue. According to some authors fibrosis could indicate a recovery (25). Instead, others described it as a poor prognostic sign (30)

- Lymphadenopathy is an inflammation of mediastinal lymph nodes. Although rare, it is reported in more severe pneumonia or in bacterial superinfection (28)

- Other rarely associated findings are nodules, small regular or irregular opacity (25), halo sign, a nodule encircled by hazy area (32), and subpleural curvilinear line (29).

Pan et al. correlated the typical CT findings with the stage of the disease (30).

1. Early stage: it occurs in the first 4 days after the onset of the disease and the typical CT finding is GGO. It can be unilateral or bilateral in subpleural localization, and it is more frequently described in the lower lobes;

2. Progressive stage: between 5 and 8 days after the onset of the pneumonia, it is characterized by the extension of GGO and by the appearance of crazy paving pattern and consolidation;

3. Peak stage: it represents the evolution of the previous stage, and consolidation became the main CT finding;

4. Absorption stage: it occurs more than 2 weeks after the onset of the infection. The patient moves toward recovery and the findings listed above disappear with the exception of GGO.

\section{LUS FINDINGS}

In order to limit the subjectivity of the exam and to obtain comparable data, Soldati et al. described a standardized protocol 
(33). It requires the examiner to analyze 14 intercostal regions for 10 seconds, with the focal point set on the pleural line. A portable convex probe $(3.5 \mathrm{mHz})$, connected wirelessly with a tablet, should be used. A first operator performs the examination, while a second operator placed at a safe distance takes care of the image management (34). In fact, despite the processing of lower quality images compared to other devices, this mode is more easily performed in the current epidemic scenario. It allows to perform the exam bedside, avoiding the movement of unstable patients, and protects operators from possible contagion using disposable plastic covers for the device, preventing any subsequent spread of the outbreak (35).

Typical LUS findings can be found in all lung fields, although bilateral posterior/lateral ones are more frequently involved (36) (Figures 3, 4):

- B-lines: vertical artifacts generated by the variation of the acoustic impedance due to the inflammatory process $(18,37)$; typically, vertical artifacts in COVID-19 patients are long,

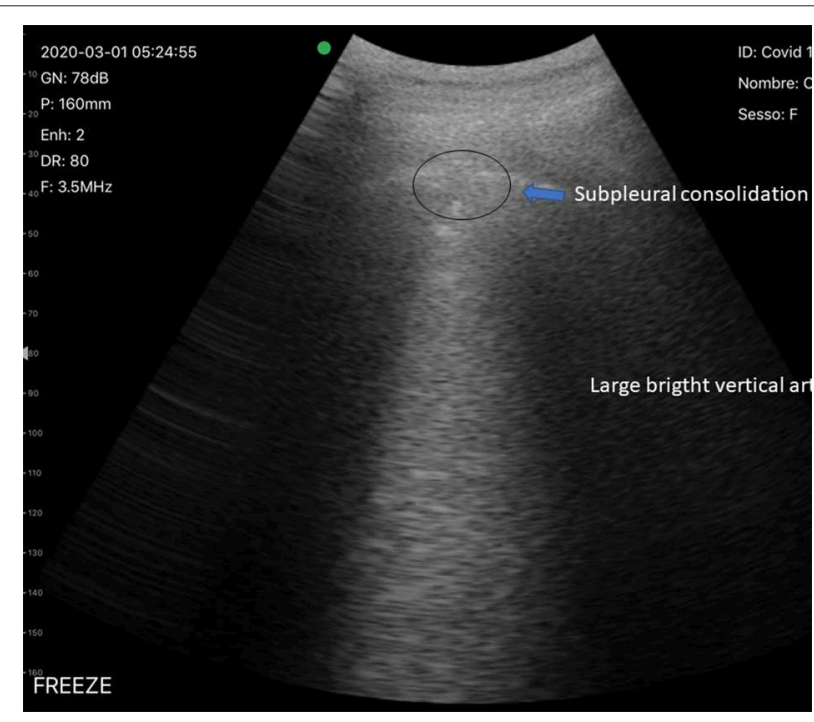

FIGURE 3 | Typical LUS findings in patients with COVID-19 pneumonia. Subpleural consolidation and vertical artifact.

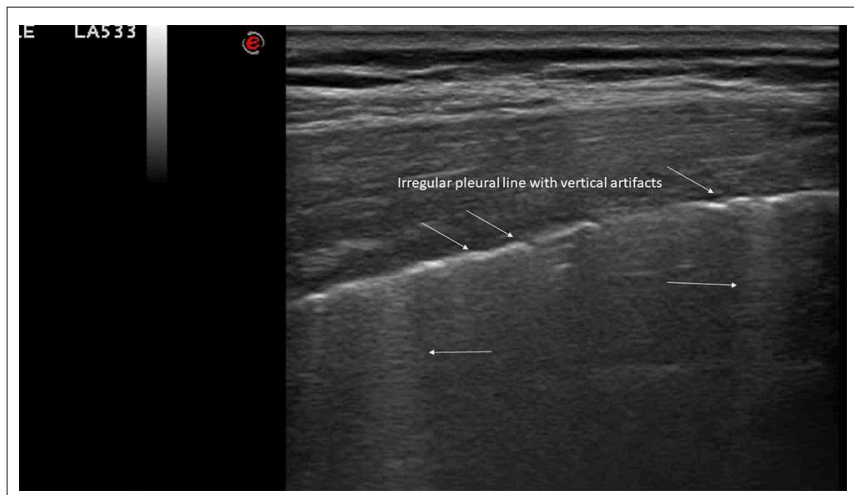

FIGURE 4 | Typical LUS findings in patients with COVID-19 pneumonia. Irregular pleural line with vertical artifacts. touch the bottom of the ultrasound screen, and are bright and thick;

- White lung: regions of white areas with the absence of Alines (horizontal and hyperechoic lines due to the normal reflection of the ultrasound beam) and vertical artifacts, which correspond to increased density of the lung parenchyma (18);

- Subpleural consolidations: irregular hypoechoic areas, indicating a collapsed lung or atelectasis $(18,37)$;

- Pleural line irregularities, such as thickening or interruptions, caused by the replacement of air with blood, pus, and fibrin according to Huang (36);

- Air bronchograms and pleural effusions are very rare and unusual, and their presence should first let the clinician thinking other diagnosis or superinfections (38).

LUS findings are related to the extent of lung injury (39). In the early stages, the lesions described are irregular vertical artifacts (B-lines) with small regions of white lung. In the intermediate stages, these lesions extend over a larger lung surface. In case of respiratory failure, subpleural consolidations are reported in a gravitational position associated with air bronchograms and large regions of white lung $(36,39,40)$. Furthermore, the diagnostic efficacy of LUS is high especially for severe patients (41).

Consequently, in order to allow comparing the severity of COVID-19 pneumonia of different patients, limiting the subjectivity and the operator-dependence of the exam, Soldati et al. proposed a LUS Score of Severity of COVID-19 Related Findings (33):

- Score 0: normal LUS pattern characterized by regular pleural line and A-lines;

- Score 1: vertical artifacts are described. The pleural line appears indented with several B-lines;

- Score 2: a broken pleural line with dark and white consolidation areas are described;

- Score 3: large regions of white lung.

However, LUS cannot be considered the gold standard for diagnosis. A limitation is that this exam cannot describe the deep lung abnormalities, since ultrasound is blocked by the presence of air. Conversely, LUS is very sensitive in detecting in small peripheral lesions and pleural effusion (36).

The typical LUS pattern of COVID-19 pneumonia is the patchy and bilateral distribution of the main lesions (42). In agreement with a group of international experts (43), during the current epidemiological scenario, the described LUS patterns in the context of fever and/or respiratory symptoms, reduced lymphocytes, and increased levels of protein C-reactive, LDH, and ferritin, are suggestive of COVID-19 pneumonia.

\section{DISCUSSION}

Six months after the first description of COVID-19 in China, the pandemic is still ongoing, and several countries are still facing the peak of the disease. Although there are still several questions to be answered regarding SARS-CoV-2 infection, the role of imaging in this pandemic is fundamental. While nasopharyngeal swabs can only diagnose the infection, CT scanning and LUS are 
both necessary to diagnose disease (COVID-19), even in case of negative microbiological results, since up to $30 \%$ of COVID-19 cases have false negative nasopharyngeal swabs. Both CT and LUS have high sensitivity to diagnose COVID-19 pneumonia and each of them has specific advantages. CT scanning is the gold standard and can easily diagnose also COVID-19 related complications, including thrombotic-hemorrhagic events, not rare in COVID19 patients. LUS is easy to perform, can be performed at bedside or even at home, and would be a feasible option also in low to middle-income countries (44). Both tests can be used as triage tools to assess those with pathological findings that would need hospitalization, allowing a proper use of the limited resources of the health systems worldwide. For these reasons, every healthcare workers should be aware of the main CT and LUS patterns of COVID-19.

\section{CONCLUSION}

In our opinion, the use of LUS during the COVID-19 outbreak has many advantages over CT, such as the bedside execution, the need for fewer operators, and the possibility of performing it at home and thus avoiding hospitalization of patients and overcrowding of the hospital. It is also less expensive (therefore easier to obtain in a developing country) and does not use ionizing radiation. Also, LUS can be used to monitor patients requiring serial examinations and in the evaluation of pregnant women, since it avoids exposure of the fetus to radiation (45).

It is important to highlight that CT and LUS are not competitive but rather complementary tools that can be used in different settings to answer different clinical questions. CT scan offers a better and comprehensive view of the lung and can also help identify complications such as infarction, embolism, emphysema; therefore, CT scanning is always helpful in case

\section{REFERENCES}

1. Zhu N, Zhang D, Wang W, Li X, Yang B, Song J, et al. A novel coronavirus from patients with pneumonia in China, 2019. N Engl J Med. (2020) 382:72733.doi: 10.1056/NEJMoa2001017

2. World Health Organization. Coronavirus disease 2019 (COVID-19) situation report-79. Geneva: World Health Organization (2020). Available online at: https://www.who.int/docs/default-source/coronaviruse/situation-reports/ 20200408-sitrep-79-covid-19.pdf?sfvrsn=4796b143_4 (accessed April 09, 2020)

3. Wang $\mathrm{H}$, Wang $\mathrm{Z}$, Dong $\mathrm{Y}$, Chang $\mathrm{R}, \mathrm{Xu} \mathrm{C}, \mathrm{Yu} \mathrm{X}$, et al. Phase-adjusted estimation of the number of coronavirus disease 2019 cases in Wuhan, China. Cell Discov. (2020) 6:10. doi: 10.1038/s41421-020-0148-0

4. Xu XW, Wu XX, Jiang XG, Xu KJ, Ying LJ, Ma CL, et al. Clinical findings in a group of patients infected with the 2019 novel coronavirus (SARSCov2) outside of Wuhan, China: retrospective case series. BMJ. (2020) 368:m606. doi: 10.1136/bmj.m606

5. Zhang JJ, Dong X, Cao YY, Yuan YD, Yang YB, Yan YQ, et al. Clinical characteristics of 140 patients infected with SARS-CoV-2 in Wuhan, China. Allergy. (2020). doi: 10.1111/all.14238. [Epub ahead of print].

6. Yang X, Yu Y, Xu J, Shu H, Xia J, Liu H, et al. Clinical course and outcomes of critically ill patients with SARS-CoV-2 pneumonia in Wuhan, China: a single-centered, retrospective, observational study. Lancet Respir Med. (2020) 8:475-81. doi: 10.1016/S2213-2600(20)30079-5 of sudden worsening of clinical conditions or for an initial assessment of moderate to severe patients if feasible in the setting where the patient is evaluated. Conversely, LUS can be used as a first level exam during the first evaluation in the emergency department or even at home to distinguish lowrisk from high-risk patients, as these would need second level exams or admission/discharge. It is useful for detecting small peripheral lesions and pleural effusion. In addition, pregnant women and children should be evaluated by LUS unless CT is considered necessary. Importantly, LUS should be preferred for follow-up and daily monitoring. Important aspects that need to be clarified are the sensitivity, the positive predictive value and the negative predictive value of the exam. Furthermore, the sharing of information and data on online platforms is essential in order to create an algorithm able to identify the typical CT and LUS findings of COVID-19 pneumonia, as already suggested by the researchers of the Italian Academy of Thoracic Ultrasound (ADET) (33).

\section{AUTHOR CONTRIBUTIONS}

$\mathrm{DP}$ and $\mathrm{DB}$ contributed conception and design of the paper and wrote the manuscript. PV, CD, RD, and RM collected the data and the articles. All authors contributed to manuscript revision, read and approved the submitted version.

\section{ACKNOWLEDGMENTS}

We wish to express our gratitude to the Italian Academy of Thoracic Ultrasound (ADET, Accademia Di Ecografia Toracica) that provided training, knowledge, inspiration, advice and telematics counseling during the COVID-19 pandemic to all Italian clinicians involved in the COVID-19 forefront.

7. Cossarizza A, De Biasi S, Guaraldi G, Girardis M, Mussini C. SARS-CoV2, the Virus that Causes COVID-19: Cytometry and the New Challenge for Global Health. Cytometry A. (2020) 97:340-3. doi: 10.1002/cyto.a. 24002

8. Zhu GD, Cao J. Challenges and countermeasures on Chinese malaria elimination programme during the coronavirus disease 2019 (COVID-19) outbreak. Zhongguo Xue Xi Chong Bing Fang Zhi Za Zhi. (2020) 32:79. doi: 10.16250/j.32.1374.2020036

9. Ai T, Yang Z, Hou H, Zhan C, Chen C, Lv W, et al. Correlation of chest CT and RT-PCR testing in coronavirus disease 2019 (COVID-19) in China: a report of 1014 cases. Radiology. (2020) 200642. doi: 10.1148/radiol.2020200642

10. Xie X, Zhong Z, Zhao W, Zheng C, Wang F, Liu J. Chest CT for typical 2019nCoV pneumonia: relationship to negative RT-PCR testing. Radiology. (2020) 200343. doi: 10.1148/radiol.2020200343

11. Huang $P$, Liu $T$, Huang $L$, Liu $H$, Lei $M$, Xu $W$, et al. Use of chest CT in combination with negative RT-PCR assay for the 2019 novel coronavirus but high clinical suspicion. Radiology. (2020) 295:223. doi: 10.1148/radiol.2020200330

12. Fang $\mathrm{Y}$, Zhang $\mathrm{H}$, Xie J, Lin M, Ying L, Pang $\mathrm{P}$, et al. Sensitivity of chest CT for COVID-19: comparison to RT-PCR. Radiology. (2020) 200432. doi: 10.1148/radiol.2020200432

13. Raptis CA, Hammer MM, Short RG, Shah H, Bhalla S, Bierhals AJ, et al. Chest CT and coronavirus disease (COVID-19): a critical review of the literature to date. AJR Am J Roentgenol. (2020) 1-4. doi: 10.2214/AJR.20.23202 
14. Bernheim A, Mei X, Huang M, Yang Y, Fayad ZA, Zhang N, et al. Chest CT findings in coronavirus disease-19 (COVID-19): relationship to duration of infection. Radiology. (2020) 295:202-7. doi: 10.1148/radiol.2020200463

15. National Health Commission of the People's Republic of China. The diagnostic and treatment protocol of COVID-19 China. (2020). Available online at: http://www.gov.cn/zhengce/zhengceku/2020-02/19/content_5480948.htm (accessed March 3, 2020).

16. Mojoli F, Bouhemad B, Mongodi S, Lichtenstein D. Lung ultrasound for critically Ill patients. Am J Respir Crit Care Med. (2019) 199:70114. doi: 10.1164/rccm.201802-0236CI

17. Mayo PH, Copetti R, Feller-Kopman D, Mathis E, Maury E, Mongodi S, et al. Thoracic ultrasonography: a narrative review. Intensive Care Med. (2019) 45:1200-11. doi: 10.1007/s00134-019-05725-8

18. Soldati G, Demi M, Smargiassi A, Inchingolo R, Demi L. The role of ultrasound lung artifacts in the diagnosis of respiratory diseases. Expert Rev Respir Med. (2019) 13:163-72. doi: 10.1080/17476348.2019.1565997

19. Demi M, Prediletto R, Soldati G, Demi L. Physical mechanisms providing clinical information from ultrasound lung images: hypotheses and early confirmations. IEEE Trans Ultrason Ferroelectr Freq Control. (2020) 67:61223. doi: 10.1109/TUFFC.2019.2949597

20. Inchingolo R, Smargiassi A, Mormile F, Marra R, De Carolis S, Lanzone A, et al. Look at the lung: can chest ultrasonography be useful in pregnancy? Multidiscip Respir Med. (2014) 9:32. doi: 10.1186/2049-6958-9-32

21. Shi H, Han X, Jiang N, Cao Y, Alwalid O, Gu J, et al. Radiological findings from 81 patients with COVID-19 pneumonia in Wuhan, China: a descriptive study. Lancet Infect Dis. (2020) 20:425-34. doi: 10.1016/S1473-3099(20)30086-4

22. Wang D, Hu B, Hu C, Zhu F, Liu X, Zhang J, et al. Clinical characteristics of 138 hospitalized patients with 2019 novel coronavirus-infected pneumonia in Wuhan, China. JAMA. (2020) 323:1061-9. doi: 10.1001/jama.2020.1585

23. Chung M, Bernheim A, Mei X, Zhang N, Huang M, Zeng X et al. CT imaging features of 2019 novel coronavirus (2019-nCoV). Radiology. (2020) 295:202-7. doi: 10.1148/radiol.2020200230

24. Hansell DM, Bankier AA, MacMahon H, McLoud TC, Muller NL, Remy J. Fleischner society: glossary of terms for thoracic imaging. Radiology. (2008) 246:697-722. doi: 10.1148/radiol.2462070712

25. Pan Y, Guan H, Zhou S, Wang Y, Li Q, Zhu T, et al. Initial CT findings and temporal changes in patients with the novel coronavirus pneumonia (2019-nCoV): a study of 63 patients in Wuhan, China. Eur Radiol. (2020) 30:3306-9. doi: 10.1007/s00330-020-06731-x

26. Song F, Shi N, Shan F, Zhang Z, Shen J, Lu H, et al. Emerging coronavirus 2019-nCoV pneumonia. Radiology. (2020) 295:2107. doi: 10.1148/radiol.2020200274

27. Ng MY, Lee EY, Yang J, Yang F, Li X, Wang H, et al. Imaging profile of the COVID-19 infection: radiologic findings and literature review. Radiology. 2:e200034. doi: 10.1148/ryct.2020200034

28. Li K, Wu J, Wu F, Guo D, Chen L, Fang Z, et al. The clinical and chest CT features associated with severe and critical COVID-19 pneumonia. Invest Radiol. (2020) 55:327-31. doi: 10.1097/RLI.0000000000000672

29. Wu J, Wu X, Zeng W, Guo D, Fang Z, Chem L, et al. Chest CT findings in patients with corona virus disease 2019 and its relationship with clinical features. Invest Radiol. (2020) 55:257-61. doi: 10.1097/RLI.00000000000 00670

30. Pan F, Ye T, Sun P, Gui S, Liang B, Li L, et al. Time course of lung changes at chest CT during recovery from coronavirus disease 2019 (COVID-19). Radiology. (2020) 295:715-21. doi: 10.1148/radiol.2020200370

31. Liu Q, Wang RS, Qu GQ, Wang YY, Liu P, Zhu YZ, et al. Gross examination report of a COVID-19 death autopsy. Fa Yi Xue Za Zhi. (2020) 36:21-3. doi: 10.12116/j.issn.1004-5619.2020.01.005

32. Li X, Zeng X, Liu B, Yu Y. COVID-19 infection presenting with CT halo sign. Radiol Cardiothorac Imaging. (2020) 2:e200026. doi: 10.1148/ryct.2020200026
33. Soldati G, Smargiassi A, Inchingolo $\mathrm{R}$, Buonsenso $\mathrm{D}$, Perrone $\mathrm{T}$, Briganti DF, et al. Proposal for international standardization of the use of lung ultrasound for COVID-19 patients; a simple, quantitative, reproducible method. J Ultrasound Med. (2020). doi: 10.1002/jum. 15285

34. Buonsenso D, Piano A, Raffaelli F, Bonadia N, de Gaetano Donati K, Franceschi F. Point-of-Care Lung Ultrasound findings in novel coronavirus disease-19 pnemoniae: a case report and potential applications during COVID-19 outbreak. Eur Rev Med Pharmacol Sci. (2020) 24:277680. doi: 10.26355/eurrev_202003_20549

35. Buonsenso D, Pata D, Chiaretti A. COVID-19 outbreak: less stethoscope, more ultrasound. Lancet Respiratory Med. (2020) 8:e27. doi: 10.1016/S2213-2600(20)30120-X

36. Huang Y, Wang S, Liu Y, Zhang Y, Zheng C, Zheng Y, et al. A Preliminary Study on the Ultrasonic Manifestations of Peripulmonary Lesions of Noncritical Novel Coronavirus Pneumonia (COVID-19). (2020). Available online at: https://ssrn.com/abstract $=3544750$

37. Demi L, van Hoeve W, van Sloun RJG, Soldati G, Demi M. Determination of a potential quantitative measure of the state of the lung using lung ultrasound spectroscopy. Sci Rep. (2017) 7:12746. doi: 10.1038/s41598-017-13078-9

38. Brogi E, Gargani L, Bignami E, Barbariol F, Marra A, Forfori F, et al. Thoracic ultrasound for pleural effusion in the intensive care unit: a narrative review from diagnosis to treatment. Crit Care. (2017). 21:325. doi: 10.1186/s13054-017-1897-5

39. Peng QY, Wang XT, Zhang LN. Findings of lung ultrasonography of novel corona virus pneumonia during the 2019-2020 epidemic. Intensive Care Med. (2020) 46:849-50.doi: 10.1007/s00134-020-05996-6

40. Poggiali E, Dacrema A, Bastoni D, Tinelli V, Demichele E, Mateo Ramos $\mathrm{P}$, et al. Can Lung US Help Critical Care Clinicians in the Early Diagnosis of Novel Coronavirus (COVID-19) Pneumonia? Radiology. (2020) 295:E6. doi: 10.1148/radiol.2020200847

41. Lu W, Zhang S, Chen B, Chen J, Xian J, Lin Y, et al. A Clinical Study of Noninvasive Assessment of Lung Lesions in Patients with Coronavirus Disease-19 (COVID-19) by Bedside Ultrasound. Ultraschall Med. (2020) 41:300-7. doi: 10.1055/a-1154-8795

42. Volpicelli G, Gargani L. Sonographic signs and patterns of COVID-19 pneumonia. Ultrasound J. (2020) 12:22. doi: 10.1186/s13089-020-00171-w

43. Piscaglia F, Stefanini F, Cantisani V, Sidhu PS, Barr R, Berzigotti A, et al. Benefits, Open questions and Challenges of the use of Ultrasound in the COVID-19 pandemic era. The views of a panel of worldwide international experts. Ultraschall Med. (2020) 41:228-36. doi: 10.1055/a-1149-9872

44. Antúnez-Montes OY, Buonsenso D. Routine use of point-of-care lung ultrasound during the COVID-19 pandemic. Med Intensiva. (2020). doi: 10.1016/j.medin.2020.04.010. [Epub ahead of print].

45. Moro F, Buonsenso D, Moruzzi MC, Inchingolo R, Smargiassi A, Demi $\mathrm{L}$, et al. How to perform lung ultrasound in pregnant women with suspected COVID-19 infection. Ultrasound Obstet Gynecol. (2020) 55:5938. doi: 10.1002/uog. 22028

Conflict of Interest: The authors declare that the research was conducted in the absence of any commercial or financial relationships that could be construed as a potential conflict of interest.

Copyright (c) 2020 Pata, Valentini, De Rose, De Santis, Morello and Buonsenso. This is an open-access article distributed under the terms of the Creative Commons Attribution License (CC BY). The use, distribution or reproduction in other forums is permitted, provided the original author(s) and the copyright owner(s) are credited and that the original publication in this journal is cited, in accordance with accepted academic practice. No use, distribution or reproduction is permitted which does not comply with these terms. 